\title{
El imperativo de acoplamiento entre sistema y mundo de la vida en Jürgen Habermas a propósito de su crítica a Talcott Parsons
}

Recibido: 21 de febrero, 2021.

Aceptado: 27 de abril, 2021.

Por: M.Ph. Ronulfo Vargas Campos ${ }^{1}$, Universidad de Costa Rica, Costa

Rica, ORCID: 0000-0002-5454-0630.

\section{Resumen}

El artículo aborda analíticamente la lectura crítica que hace Jurgen Habermas de la sociología funcionalista de Talcott Parsons a la luz de las preocupaciones habermasianas por procurar una teoría social que dé cuenta sustancial de las interacciones intersubjetivas al interior de las estructuras o sistemas sociales. A Habermas le concierne particularmente el desarrollo de un corpus conceptual que resuelva la controversia entre la acción social autónoma y las pautas conductuales emanadas de entramados institucionales que tienden a orientar la interacción en dirección al imperativo de autoconservación y en detrimento del imperativo de la vida buena en el contexto de la Lebenswelt. En tal sentido, el esfuerzo de Habermas por criticar la sociología parsonsiana a la vez que integrar sus elementos en una teoría social más comprensiva se inscribe en el entendimiento de la Teoría Crítica como saber emancipatorio. El autor de este artículo suscribe ese entendimiento, y su aporte pretende mantener a la vista una problemática que no ha dejado de tener actualidad en un mundo contemporáneo globalizado bajo las condiciones de la organización sistémica y sus consecuencias conflictivas para la ciudadanía.

\section{Abstract \\ The system-lifeworld coupling imperative in Jürgen Habermas apropos his critique of Talcott Parsons}

This article is an analytical review of Jürgen Habermas' critical reading of Talcott Parsons' functionalist sociology, stemming from Habermas' concerns for a social theory to provide a substantial account of intersubjective interactions within social structures or systems. Habermas is particularly concerned with the development of a conceptual corpus to resolve the controversy between autonomous social action and the behavioral patterns emanating from institutional frameworks that tend to move social interaction toward self-preservation as an imperative, while simultaneously in detriment of Lebenswelt's imperative of the good life. In this sense, Habermas' effort to criticize Parsonian sociology while integrating its elements into a more comprehensive social theory is inscribed in the understanding of Critical Theory as emancipatory knowledge. The author of this article subscribes to this understanding, aiming to uphold the view of a lingering issue in a contemporary globalized world under the conditions of systemic organization and its conflictive consequences for citizenship.

1 El profesor Ronulfo Vargas Campos se graduó de la Maestría en Filosofía de la Universidad de Costa Rica, Costa Rica, y es doctorando del programa Literaturas de Expresión Española en la Université Laval, Canadá. Tiene más de 20 años de trabajar como docente en la Escuela de Estudios Generales de la Universidad de Costa Rica. Contacto: ronulfovargas@ yahoo.com.

PALABRAS CLAVE:

ideologías, filosofía, sociología, Teoría Social sociedad, vida

\section{KEY WORDS:}

Ideology, philosophy, sociology, social theory, society, life 
Este artículo aborda lo que Jürgen Habermas considera el problema del desacoplamiento entre el sistema y el mundo de la vida, que son constelaciones conductuales que responden a fines diversos pero complementarios. No obstante, difieren, también en los medios implementados para sus respectivos fines. En el caso del sistema, la acción racional con arreglo a fines, un concepto acuñado en la teoría de la acción de Max Weber y que Max Horkheimer (1973) interpretó como razón instrumental. En el caso del mundo de la vida, la comprensión (Verstehen) del sentido, fórmula provista por Edmund Husserl, así como la acción racional con arreglo a valores, en expresión de Weber, o racionalidad sustantiva, según Horkheimer (1973). Toda esta problemática redunda en la cuestión de la sostenibilidad de una socialización que privilegia la eficacia en la consecución de fines estructurales -la autoconservación- y descuida el sentido y la calidad de la vida humana.

En la historia de la filosofía el eclecticismo denomina una tendencia de la Academia platónica en su tercera etapa, hacia el siglo II a.C., que procuraba sintetizar doctrinalmente aportes de los más importantes sistemas de pensamiento de la época: Platón, Aristóteles, Epicuro y Zenón de Citio (Ferrater-Mora, 1998). Las doctrinas eclécticas no pretendían ser una mezcla heterogénea e inconexa de ideas, sino una sabiduría que articulara armoniosamente nociones de diversas fuentes mediante el recurso de la elección (eklektós, el término griego de donde deriva el nombre eclecticismo, admite acepciones tales como elegido, escogido, distinguido, notorio, lo cual permite intuir que en la elección eclética se produce a la vez la discriminación de todo lo que no se considera de importancia), que implicaba naturalmente crítica y discriminación de aquello que no encajara en el marco en desarrollo. Por tanto, la palabra eclecticismo ha servido desde entonces para referirse a todo intento teórico que se desempeña en esos términos: seleccionando tras analizar e integrar ideas valiosas de diversos intelectuales.

Jürgen Habermas, uno de los pensadores contemporáneos más importantes, puede ser identificado desde múltiples determinaciones. Por ejemplo, pertenece a la segunda generación de la Escuela de
Frankfurt; realizó esfuerzos sistemáticos por integrar una teoría de la acción comunicativa, con un talante más propositivo, aunque no menos crítico, que el de sus predecesores, los teóricos del Gran Rechazo; mantuvo una determinante polémica con la filosofía posmodernista; y su prolongada lectura crítica de la sociología funcionalista en autores como Parsons y Luhmann. Por ende, es un ecléctico.

Al respecto, Axel Honneth, en su artículo sobre la Teoría Crítica de la obra colectiva La teoría social hoy (Giddens et al., 1990), centra su crítica en el funcionalismo de esta escuela contemporánea, que, al igual que los eclécticos clásicos, se decantó por integrar una serie de corrientes de pensamiento: Kant, Hegel, Marx, Durkheim, Weber, entre otros. En este sentido, Habermas es un continuador de ese esfuerzo por integrar críticamente elementos de otros intelectuales y escuelas de pensamiento. La teoría weberiana de la acción, las consideraciones del giro lingüístico, la teoría del derecho, y también, el funcionalismo y la teoría de sistemas de la sociología de Parsons y Luhmann, sobre la que deseamos centrarnos en este artículo. Se debe mencionar que puede considerarse funcionalista a la Escuela de Frankfurt, y por extensión a Habermas, porque lo son en el sentido crítico integrador que ha sido su praxis teórica habitual.

En torno al funcionalismo inaugurado por Talcott Parsons, Habermas reconoce su estatus de teórico insoslayable para cualquier investigador de la sociedad. Algunas de las consideraciones para el respeto son: 50 años de desarrollo sistemático de la teoría social, el esfuerzo sostenido de integrar elementos de otras teorías a la propia, el aprecio que le merecieron esas teorías, la generalidad de pretensiones omniabarcadoras del estructural funcionalismo parsonsiano. No obstante, esa misma generalidad le induce a Habermas la impresión de que su teoría llega a apartarse de su objeto intrínseco, la sociedad, para devenir en concepción de mundo.

Esta observación mencionada la hace extensiva al funcionalismo estructural del neoparsonsiano Niklas Luhmann, quien en su obra apela a la autonomía fáctica y absoluta del sistema: fáctica y absolutamente independiente de factores huma- 
nos. Los sistemas disponen su funcionamiento sin intervención de la consciencia humana debido a que hacen clausura a su entorno y desarrollan autorreferencialidad y autogeneración (Luhmann, 2010; Habermas, 1993). Sobre ello, Izuzquiza (2008) dedica una obra monográfica a este teórico, elocuentemente titulada La sociedad sin hombres, en la que destaca esta alienación sistémica.

El prurito integrador será, además, la razón de la primera observación crítica de Habermas, porque la integración forzada de teorías difícilmente compatibles desemboca en un pseudo-eclecticismo que se resiente por incoherencias — recordemos que nos hemos referido al propio Habermas como un ecléctico que asigna el mayor valor a la coherencia de lo integrado- . En el funcionalismo de Parsons los problemas empiezan cuando intenta articular a su teoría de la acción, que surge desde el idealismo neokantiano de Durkheim y Weber, el paradigma de la teoría de sistemas que Habermas juzga positivista. Con ello, Ilegan a coexistir en el estructural funcionalismo parsonsiano dos formas de integración que tienden a repelerse: la integración social y la integración funcional. La primera se produce desde acciones de agentes orientadas al entendimiento (Verstehen), que tiene como trasfondo el mundo de la vida; la segunda, desde funciones estructurales que obliteran el entendimiento, porque se ejecutan mediante emisión de directrices.

La diferenciación del sistema general de la acción en subsistemas autonómicos es la raíz de los problemas de coherencia, puesto que el sistema general se postula como un conglomerado de subsistemas dotados de mecanismos propios. La diferenciación parsonsiana entre el sistema de la personalidad y el sistema social es ejemplar como fuente de contradicciones:

Los mecanismos de la personalidad como sistema no son los mismos que los mecanismos del sistema social, por razón de que, como ya hemos señalado en el presente estudio y en algunas otras ocasiones, las personalidades y los sistemas sociales constituyen dos clases diferentes de sistemas. En tanto en cuanto la «psicología» nos da unas leyes muy generalizadas res- pecto al proceso de la motivación, estas serán tan aplicables directamente a los procesos de acción en el contexto del sistema social como a cualquier otro aspecto. Pero en tanto en cuanto la psicología no nos proporcione leyes, sino mecanismos, todas las probabilidades son de que se trate de mecanismos de la personalidad como sistema (Parsons, 1984, p. 132).

Al tratar de conservar la teoría de la acción para el sistema de la personalidad, incurre en contradicción porque dota a este sistema de mecanismos. Este es un concepto de la teoría de sistemas que alude a dispositivos que condicionan acciones. Esto aplicado a la personalidad causa que estemos ante una perspectiva conductista afín a la teoría de sistemas, pero incompatible con conceptos de la teoría de la acción, tales como: comprensión y sentido; puesto que la acción mecánica elude la consciencia. Su afán por autonomizar sistemas diferenciados conduce a Parsons a privilegiar el sistema social por encima del sistema de la personalidad cuando afirma que la psicología apenas provee intuiciones para explicar la motivación, un proceso que implica autoconsciencia, e, inmediatamente, afirma que lo que provee la psicología en realidad son mecanismos que regulan la personalidad. Se debe mencionar que el sistema social, coherentemente, opera mediante mecanismos estructurales: pautas conductuales, funciones, productos.

El mundo de la vida (Lebenswelt) es el concepto central que Parsons desestima y Habermas conserva en su propia teoría social integradora. En su obra de 1936, La crisis de las ciencias europeas y la fenomenología trascendental, Edmund Husserl emplea el término mundo de la vida para aludir al bagaje de experiencias sedimentadas que hace posible el sentido en toda actividad humana. Es el ámbito de la experiencia pre-tematizada, de la vivencia espontánea e inmediata que funda la subjetividad en términos de intersubjetividad: convivencia y comunicación entre sujetos formados por el bagaje experiencial que comparten. Toda concreción humana es un hecho del mundo de la vida, porque se da desde ese bagaje y viene a enriquecerlo. 
En otras palabras, el mundo de la vida no es un hecho estático, sino un horizonte de hechos que surge en la historia, se transforma y se diversifica por la experiencia que él mismo abre. La ciencia, o la actividad teorética, es hecho tematizado del mundo de la vida, que es pre-temático y pre-teorético, pero condición de posibilidad de cualquier tematización teórica. En la obra citada, Husserl trata el concepto en términos de vida experienciante que precede, hace posible y acompaña a la experiencia teorética:

El sentido de ser del mundo de la vida pre-dado es una configuración subjetiva, que resulta de la vida experienciante, pre-científica. En ella se construye el sentido y la validez de ser del mundo, y en cada caso del mundo que efectivamente vale para quien tiene la experiencia correspondiente. Por lo que concierne al mundo "objetivamente verdadero", al de la ciencia, él es la configuración de grado más alto sobre la base del experimentar y pensar científico, respectivamente, de sus producciones de validez (Husserl, 2008, p. 112).

A partir del concepto husserliano, Habermas (1999) define el vocablo mundo de la vida como el "trasfondo contextualizador de los procesos de entendimiento" (p. 288). Pizzi (2005) da cuenta específicamente de las relaciones entre Husserl y Habermas a propósito de las categorías de sentido, comprensión y mundo de la vida. El sentido preconceptual de los contenidos del mundo de la vida sirve de base para la estructuración de un orden social, sin que se precise, según Habermas, para ello un concepto técnico de sistema, porque el mundo de la vida es ya un orden estructurado:

Las certezas del mundo de la vida no solamente tienen el carácter cognitivo de tradiciones culturales habitualizadas, sino también, y por así decirlo, el carácter psíquico de competencias adquiridas y comprobadas, y el carácter más bien social de solidaridades acreditadas. La aproblematicidad del mundo de la vida en y a partir del que se actúa comunicativamente se debe no sólo a ese tipo de seguridad que deriva de lo que se sabe trivialmente, de lo que todo el mundo sabe, sino también al tipo de certeza que deriva de la conciencia de ser capaz de algo o de poder fiarse de alguien. [...] En las certezas del mundo de la vida van todavía juntos, de forma más o menos difusa, esos tres componentes: el saber cómo se hace algo y el saber en qué puede uno estribar (Habermas, 1999, p. 315).

El lenguaje es uno de los componentes esenciales del mundo de la vida, en la medida en que interpreta simbólicamente la experiencia y permite comunicarla, con lo que se produce la comunidad lingüística que se integra mediante la comunicación de contenidos de consciencia, que produce la intersubjetividad. La intersubjetividad es comunidad de contenidos de consciencia. La teoría de Parsons (1984), que reinterpreta el mundo de la vida en términos de sistema, reduce el lenguaje a un medio de control. Mediante el lenguaje, los sistemas explicitan sus funciones. La comunicación lingüística al interior de los sistemas se emprende como acción racional con arreglo a fines, por mor de la maximización de la eficiencia. Es una comunicación de "orientaciones requeridas" (Parsons, 1984, p. 135) para el funcionamiento eficiente del sistema.

El funcionamiento sistémico se optimiza autorregulándose mediante la automatización de sus funciones, para lo cual prescinde de la comunicación lingüística orientada al entendimiento. Tratándose de "orientaciones requeridas", cualquier conducta que no responda a ellas se interpretará como desviación de expectativas de roles e, ipso facto, tendrá que enmendarse:

Un mecanismo de control social es un proceso de motivación en uno o más actores individuales que tiende a reaccionar frente a una tendencia a la desviación en el desempeño de las expectativas de rol, en él mismo o en uno o más alter. Se trata de un mecanismo de restauración del equilibrio (Parsons, 1984, p. 135).

La reacción a la desviación o infracción a directrices en el marco del funcionamiento sistémico se produce como enmienda a través de sanción o castigo al infractor.

Habermas convendrá en que la comunicación sistémica, reducida a la función lingüística directiva, es pertinente en contextos donde se minimiza la 
posibilidad de diálogo. Verbigracia, en los que se dirimen cuestiones eminentemente técnicas, en los que el cuestionamiento de directrices sea ocioso y entorpezca fines, como puede ser la comunicación entre el mecánico y su ayudante ante un vehículo en reparación.

Asimismo, Habermas cree que el mundo de la vida es el trasfondo que posibilita toda acción y, por ende, también facilita la estructuración de sistemas: los sistemas surgen en el mundo de la vida. Sin embargo, el tipo de acción sistémica entra en contradicción con la acción que se emprende espontáneamente en el marco del mundo de la vida. La comunicación lingüística es un hecho del mundo de la vida y, antes que coordinar por directrices inapelables, procura espontáneamente la interacción lingüística basada en el intercambio de argumentos con pretensiones de validez: cada interlocutor pretende naturalmente que su argumento exprese contenidos 1) inteligibles, 2) verdaderos, 3) veraces o sinceros, y 4) correcta o adecuadamente expresados (Habermas, 1999). Si estas pretensiones no se cumplen -si los argumentos se muestran ininteligibles, falsos, mendaces, o incorrectamente formulados-, no hay comunicación, por obstrucción de patologías lingüísticas.

En la vida cotidiana, normalmente, la comunicación orientada al entendimiento satisface pretensiones de validez, en tanto que los interlocutores se interpelan con argumentos cuyo análisis revela la presencia de las pretensiones. La eficiencia, entendida como procuración de la inmediatez, no es un objetivo de la comunicación lingüística orientada al entendimiento. La razón comunicativa discurre analíticamente, esto es, mediatizando los contenidos que aprehende y juzgando si reúnen pretensiones de validez.

En cambio, la razón funcional pretende el acatamiento inmediato a las funciones. Desde la razón comunicativa que arraiga en el mundo de la vida pueden incluso cuestionarse las pretensiones sistémicas funcionales, pero no a la inversa. El mundo de la vida puede explicar los sistemas, pero estos no hacen lo propio con aquél.
La teoría de la comunicación que Parsons utiliza es insuficiente para comprender la complejidad del fenómeno de la comunicación humana. Parsons representa este fenómeno bajo la categoría de la doble contingencia: ego y alter interactúan esperando algo uno del otro. Pero como ambos son libres, entonces puede pasar cualquier cosa. Por eso uno y otro intentarán condicionar la libertad ajena: "Toda decisión es contingente, podría haberse tomado una decisión distinta. De ahí que ego y alter tengan que tratar de condicionar la libertad del otro para que las decisiones del otro no redunden en detrimento de los propios intereses" (Habermas, 1999, p. 396).

Parsons define dos clases de medios o estrategias a las que apelan los actores para influirse, para hacer prevalecer intereses particulares: estrategias positivas (convencimiento o incitación por dinero) y negativas (admonición moral o intimidación sustentada en un poder superior). Ego decide, con arreglo a las circunstancias, si emite información verdadera o la distorsiona, para convencer a alter mediante engaño, o si apela directamente a su ambición. También decide si lo constriñe, manipulando su consciencia moral; o abiertamente lo reprime, para hacer prevalecer intereses particulares.

La acción puede estar orientada hacia el logro de una meta, la cual es un estado futuro de las cosas anticipado; se siente que promete gratificación el alcanzarla; un estado de las cosas que no se logrará sin la intervención del actor en el curso de los sucesos. Esa orientación instrumental o hacia una meta introduce un elemento de disciplina: la renunciación a ciertas gratificaciones inmediatamente potenciales, incluyendo las que pueden derivarse de «dejar que las cosas pasen» pasivamente y esperar el resultado. Se renuncia a esas gratificaciones inmediatas en interés de mayores ganancias posibles que se deriven de alcanzar la meta; logro que se siente que se relaciona con el cumplimiento de ciertas condiciones en los estadios intermedios del proceso (Parsons, 1984, p. 34).

Por un lado, cuando apela al dinero o al poder, ego se comporta orientándose hacia su propio éxito y 
su acción está orientada por la racionalidad instrumental. Por otro lado, cuando apela al convencimiento, ego se orienta al entendimiento. Entonces, dinero y poder son motivaciones empíricas, mientras que el entendimiento es lógico y semántico. Si la convicción se produce mediante entendimiento basado en información verdadera, la acción de ego podría estar orientada por la racionalidad sustantiva, con arreglo a valores. No obstante, sin que esa sea la orientación definitiva, ego aún podría instrumentalizar un entendimiento obtenido con base en información verdadera.

Lo que a mí me irrita sobre todo es la circunstancia de que en el esquema se hace subrepticiamente uso de la peculiar asimetría entre la forma estratégica y la forma consensual de ejercer un influjo, a la vez que se la hace desaparecer mediante la hechura empirista de la conceptuación que se emplea (Habermas, 1999, p. 396).

Es interesante recordar que el estructural funcionalismo parsonsiano suele ubicarse en la tradición sociológica del consenso, que privilegia analíticamente el momento de la integración cohesiva, por procesos armoniosos de interiorización e institucionalización. Sin embargo, Parsons sitúa la comunicación en el ámbito de estrategias particulares para influir sobre la decisión de los interlocutores. Es decir, en su representación de la interacción comunicativa, los actores aparecen enfrentados desde posiciones antagónicas, que nunca abandonan. Entonces, la comunicación es una lucha para hacer prevalecer intereses particulares.

La situación presentada en la doble contingencia guarda analogías con la posición inicial de los actores de la alegoría del amo y el esclavo, que es el relato clásico que ilustra la orientación sociológica del conflicto: dos autoconsciencias enfrentadas a muerte por el reconocimiento exclusivo de la condición subjetiva. Hasta ahí la analogía, puesto que, en la narración hegeliana, la interacción de las autoconsciencias lleva al reconocimiento de la legítima subjetividad del otro como una acción imprescindible para la realización de la propia subjetividad.

La intersubjetividad parsonsiana establece una pseudocomunicación, porque cada interlocutor permanece atado a intereses particulares. Revela, además, la ausencia de una comunidad de valores y por ende el fracaso de la interiorización, porque si tuviera eficacia, los intereses particulares podrían consensuarse sobre la base de valores compartidos. La doble contingencia, en resumen, señala la inoperancia del sistema cultural.

La cultura, en efecto, parece accesoria en el marco de la teoría parsonsiana, porque las interacciones se conciben desde una preconcepción individualista de las relaciones sociales. La libertad que expresan a través de la acción remite al imperativo sistémico de autoconservación y no al imperativo cultural del deber. Es una libertad individualista, que se demuestra prácticamente en la capacidad de avasallar al otro, sin superar una estructura de dominación y sujeción. El egocentrismo de los actores es una condición insuperable, porque se los comprende, desde las categorías sistémicas, como sistemas psicosomáticos autorreferenciales, cerrados a su entorno.

En este sentido, pese a que Parsons ubica en la doble contingencia a dos agentes conscientes de sus intereses particulares, que deliberan estrategias para realizarlos, la prevalencia de la voluntad de uno u otro se hace depender del esquema conductista de reacciones inmediatas a estímulos empíricos: premios o castigos son las motivaciones que coordinan la voluntad de alter conforme a la de ego, sin que la deliberación consciente intervenga substancialmente.

La doble contingencia, en la que la interacción lingüística está mediada por la razón funcional, se presenta como modelo general de comunicación. Sin embargo, Habermas discrepa, ya que acepta que es el modelo de la comunicación sistémica, en la que la explicitación de motivos no es siquiera requisito, pues depende más bien de explicitar claramente directrices funcionales. Esto debido a que el propósito de la comunicación no es el entendimiento, sino la función. En síntesis, la eficacia del lenguaje reducido a medio de control será tanto mayor en la medida en que menos tiempo dispongan los agentes para ponderar el contenido del mensaje.

Contra esta concepción general de la interacción lingüística, Habermas argumenta que hay al menos 
otro modelo de comunicación: la acción comunicativa, que no responde a la función, sino a la comprensión o entendimiento. No está mediada por la razón formal, sino por la razón sustantiva y no apela, salvo para cancelarse a sí misma, a mecanismos conductistas de coerción.

El contexto de la acción comunicativa no es el sistema, sino el mundo de la vida, desde donde la acción se despliega cotidianamente. El propósito de la acción comunicativa es la búsqueda de consenso, que crea comunidad. Cuando los interlocutores se interpelan, en una situación normal de habla, interactúan lingüísticamente -intercambian actos de habla- con la pretensión de explicitar motivos que representan intereses. No obstante, el interés general de esa interacción es la comprensión: hacer comprender a alter los motivos de ego, y viceversa, para que entre ego y alter se produzca comprensión y reconocimiento de la legitimidad de intereses particulares. Si en efecto se producen, entonces hay consenso respecto de los intereses y estos se comparten (se generalizan).

La comunicación exitosa, que produce entendimiento y consenso, demanda que en la interacción lingüística los contenidos expliciten pretensiones de validez; es decir, que las proposiciones del discurso sean inteligibles, veraces, sinceras y que se emitan con arreglo a normas compartidas de expresión. La comunidad se establece normalmente cuando los interlocutores se comunican intercambiando pretensiones de validez.

Asimismo, la comunicación tiende a frustrarse cuando los interlocutores se interpelan mediante proposiciones ininteligibles, falsas, mendaces o distantes de una correcta emisión. En estos casos, la acción comunicativa se cancela a sí misma, porque incomunica. Sin comunicación sustantiva -con arreglo al entendimiento- no hay comunidad ni consenso, sino desagregación o imposición forzada de voluntades, por medio de la comunicación formal -con arreglo a la función-.

Para Habermas, la interacción lingüística que sigue el modelo de la acción comunicativa se produce en un espacio libre de distorsiones o no se produce. Los interlocutores se dispersan, o asumen el mode- lo de la acción funcional; pero en la cotidianeidad social extrasistémica, la acción comunicativa se expresa normalmente. En la interacción lingüística cotidiana, los interlocutores intercambian actos de habla cuyas pretensiones de validez son ponderadas y juzgadas, y si se reconocen, se produce la comunidad de consenso.

Parsons no tiene en cuenta, ni puede hacerlo en el marco de su teoría de la acción, que el concepto de sanción no puede aplicarse a las posturas de afirmación o de negación frente a pretensiones de validez susceptibles de crítica (Habermas, 1999, p. 397).

Por ende, la crítica racional de las pretensiones de validez es la única condición para que se establezca el consenso. La comprensión adviene si los interlocutores reconocen la presencia de esas pretensiones en los actos de habla de cada uno. No hay lugar, en la acción comunicativa, para premios o castigos empíricos, porque son factores distorsionadores de la comunicación sustantiva. La comunicación sistémica no es sustantiva sino instrumental, y la apelación a premios o castigos empíricos sigue una lógica conductista que evade el discernimiento, porque apuesta a reacciones inmediatas, condicionadas. La premisa de la acción comunicativa es que los interlocutores son sujetos conscientes y racionales que aspiran al entendimiento recíproco del sentido -que no es premio ni castigo empírico-, porque tienen autonomía intelectual.

La acción comunicativa contiene el potencial de producir consenso, que se incrementa cuando se la articula al discurso. Esto ocurre cuando se la traslada desde la espontaneidad preteórica del habla que se expresa en el mundo de la vida hasta el discurso teórico, premeditado y conceptualmente elaborado, que tiene lugar en contextos sistémicos.

Habermas enfatiza que, en la sociología de Parsons, el lugar del mundo de la vida lo ocupa el sistema de la cultura, que es la articulación de normas y valores que operan en el proceso de integración social. Sin embargo, el sistema cultural no engrana bien en la teoría, porque Parsons lo define por la validez, en contraste con la facticidad de los demás sistemas. El sistema cultural, según él, no es empírico sino 
semántico; es decir, son "sistemas de creencias o ideas" en un componente, y en el siguiente "sistemas de símbolos" (Parsons, 1984, p. 211), y no se expresa en la experiencia antes de la interiorización y la institucionalización.

Por la interiorización, los agentes responden al imperativo del deber por el deber, en tanto que, por la institucionalización, responden a ese imperativo conforme al deber. La fuerza del sistema cultural radica entonces en la interiorización, porque la acción conforme al deber está garantizada por el sistema jurídico-político, que es empírico, e integra funcionalmente a los agentes. Por ende, solo el sistema cultural gira en torno al imperativo del deber: los demás lo hacen en torno al imperativo de la autoconservación.

De lo anterior, se sigue que los agentes de la acción funcional devienen medios para la autoconservación de los sistemas que, como "plexos de acción" autónomos, se autoconservan estructurando la acción con arreglo a fines, en la que no interviene la consciencia de los agentes. Por este motivo, para la racionalidad funcionalista la autonomía corresponde al sistema y no a los agentes, que en realidad no son tales, porque reaccionan automáticamente a directrices funcionales. Los valores de la cultura ceden ante los imperativos de la autoconservación sistémica.

El sistema de la cultura que, según Parsons, es dominante por contener normas y valores, adquiere la forma de una declaración vacía de principios. Lo determinante, en última instancia, es la acción funcional con arreglo a fines, que son los de la autoconservación. Una evidencia de la vacuidad práctica del sistema cultural la aportan los "hechos problemáticos", que derivan de conflictos entre la acción cultural con arreglo a valores y la acción funcional con arreglo a fines.

Por ejemplo, los bombardeos sobre Hiroshima y Nagasaki representan uno de estos "hechos problemáticos", en los que la acción se resuelve a favor de la autoconservación. Por el imperativo de la autoconservación, los sistemas tienden a independizarse del sistema cultural, lo que en la práctica representa un avasallamiento de la cultura o, como lo teoriza Habermas, la colonización del mundo de la vida por el sistema.

En el ejemplo aludido, el imperativo de la autoconservación dicta el bombardeo de las ciudades japonesas, porque el sistema geopolítico lo demanda en la coyuntura del final de la Segunda Guerra Mundial, que perfila a la Unión Soviética como superpotencia militar y cultural en competencia con los Estados Unidos por la hegemonía mundial. Lo que desde el mundo de la vida parecía tener sentido pacificación, reconciliación, prevalencia del entendimiento entre naciones, en general los autoproclamados valores de la civilización- es anulado por la acción bélica que afirma con contundencia la superioridad militar ante el otrora aliado soviético.

La frecuencia de "hechos problemáticos" en la concreción de la acción social no representa para Parsons una señal de incoherencia, aun cuando patentiza el conflicto entre el deber ser y el ser, o entre validez y facticidad, de acuerdo con la concepción que la teoría da a esas dimensiones. La teoría no explica por qué, si el sistema cultural aporta normas y valores, que son interiorizados e institucionalizados, la acción final obedece al imperativo de la autoconservación, lo cual demuestra que las normas y los valores del sistema cultural son nada más nominales. Ante hechos problemáticos, el sistema cultural se vuelve disfuncional y genera conflictos.

El compromiso de Parsons con la teoría de sistemas, manteniendo las categorías de la teoría de la acción, le hace incurrir en un dualismo entre el sistema de la cultura -normativo sobre la base de la acción orientada al entendimiento -y los demás subsistemas - empíricos sobre la base de la acción funcional con arreglo a fines-. En última instancia, estos últimos son determinantes de la acción consumada, en tanto que la coordinan sin apelar al entendimiento, sino mediante directrices estructurales. Este dualismo ecléctico es causa de la incoherencia, porque: 1 ) desde la teoría de la acción, el sistema cultural no puede expresarse en sí mismo como realidad empírica; 2) desde la teoría que pretende ser complementaria, los sistemas son organizaciones funcionales dadas. Al respecto, Habermas (1999) señala: 
A lo largo de toda su obra operan dos programas diferentes: un programa "acción social" en la tradición idealista y otro "sistema social" en la tradición positivista. El programa "acción social" se centra en el significado que una acción tiene para un actor, mientras que el programa "sistema social" se centra en las consecuencias que una actividad tiene para un sistema de actividad (p. 284).

Por un lado, el sistema social, desde la teoría de sistemas, aparece, en sentido conductista, como un sistema de comportamientos que pueden describirse empíricamente e inducirse con estímulos de reacción inmediata. Por otro lado, el sistema cultural, desde la teoría de la acción, surge como el conjunto de ideales normativos de la acción: su horizonte de sentido. En Parsons coexisten en paralelo ambos paradigmas, lo que aporta ambigüedad a la teoría.

La coexistencia incoherente entre el sistema cultural normativo y los sistemas funcionales empíricos evoca la imagen de dos realidades ontológicamente distintas, con el predominio de una sobre la otra, es decir, el dualismo. Pero este es un efecto de la coexistencia de marcos teóricos poco integrados. Recuperando la categoría del mundo de la vida e interpretando el sistema cultural parsonsiano desde esta categoría, Habermas afirma que en efecto se establece un dualismo entre el mundo de la vida y el sistema, pero que no se trata de ontología, sino de "desacoplamiento":

Si empleamos un concepto de sociedad articulado en dos niveles, que incluya mundo de la vida y sistema, puede hacerse comprensible esta elaboración patógena de los conflictos que surgen entre los imperativos de la integración social y los de la integración funcional. [...] Con el concepto de mundo de la vida la esfera de las pretensiones de validez que Parsons ve ubicada en la trascendencia de unos contenidos semánticos culturales libremente flotantes, quedaría inserta de antemano en plexos de acción empíricos e identificables en el espacio y en el tiempo. [...] Con ello no desaparece del todo el dualismo entre exigencias de la cultura e imperativos de la supervivencia. Pero ese dualismo adopta una forma distinta cuando el concepto de sistema es desarrollado a partir del concepto de mundo de la vida y no se lo encaja directamente sobre el concepto de acción (Habermas, 1999, p. 330).

Desde esta perspectiva, el mundo de la vida y el sistema no son dos realidades diferentes, sino dos estructuraciones de la experiencia y la acción. Habermas consigue, de esta manera, articular o integrar la categoría de sistema, junto con el repertorio conceptual de los sistemas complejos, al marco teórico de la teoría de la acción, en el que opera la categoría de mundo de la vida. El sistema es una organización del mundo de la vida que se rearticula como estructura funcional con arreglo a fines. Solo después de rearticularse en estos términos, aparece como algo distinto al mundo de la vida.

Sin embargo, como indicaba Husserl, el mundo de la vida preexiste a sus configuraciones concretas y las acompaña para darles sentido. Cuando la configuración funcional del sistema es recalcitrante al acompañamiento del mundo de la vida, sistema y mundo de la vida aparecen como cosas distintas, porque en efecto, se reifican, y una de esas "cosas" cae sobre la otra como imposición. Esta imposición es la de la acción funcional con arreglo a fines, que opera como estímulo para reacciones inmediatas, sobre la acción racional con arreglo al valor del entendimiento entre sujetos que se interpelan.

De la interpretación de Habermas inferimos que lo que ocurre en la realidad es que el mundo de la vida produce sistemas. Los sistemas son hechos del mundo de la vida; es decir, son mundo de la vida, pero organizados de tal forma que se le enfrentan y coordinan las acciones intersubjetivas por encima del mundo de la vida que les preexiste y que normalmente coordina acciones con arreglo al entendimiento que favorece la integración social. Por ende, la consecuencia es que los sujetos dejan de ser actores del mundo de la vida para ser actuados por los sistemas, toda vez que la acción deja de estar supeditada al entendimiento. Los sistemas toman el lugar de los actores subjetivos.

Dicho proceso, la colonización del mundo de la vida por el sistema, es la forma que adquiere la 
alienación en la modernidad tardía: el sistema es mundo de la vida alienado. Esta es una perspectiva enriquecedora, porque no solo reintegra coherentemente perspectivas teóricas -que en Parsons resultaban contradictorias-, sino que lo hace críticamente, pues puntualiza los efectos prácticos, sobre los actores, su calidad de vida y su entorno, que conlleva la supeditación anormal del mundo de la vida al sistema.

La teoría parsonsiana de la evolución social -que Habermas acepta- explica la concreción de los sistemas actuales por incremento en la complejidad y diferenciación respecto a anteriores formas de organización:

In quite primitive societies, there actually is little differentiation between the general structures of a society and its religious organization. In more advanced societies, the interrelation of social and cultural systems in the religious and legitimation contexts involves highly specialized and complicated structures" (Parsons, 1966, p. 11).

El desacoplamiento entre los sistemas y los mundos de la vida responde a ese aumento en complejidad y diferenciación -especialización- de funciones. Tanto el sistema como el mundo de la vida aumentan su complejidad y diferenciación, pero en formas distintas. Para comprender las formas actuales de la evolución, Habermas recuerda el estado de sociedades premodernas, menos complejas, en las que había poca diferenciación entre las diversas actividades humanas: trabajo, familia, religión y política. Todas esas actividades eran esferas de la vida que no se disputaban espacios privilegiados, porque tenían a converger en la experiencia cotidiana, aun cuando las producciones de esas actividades fueran particulares. Es decir, los sistemas de producción, reproducción y comunicación no eran autónomos ni estaban diferenciados del mundo de la vida. El desacoplamiento del sistema y del mundo de la vida, así como la posterior colonización de este por aquel, es la forma característica en que evoluciona la sociedad moderna. Tal como lo señala Habermas (1999):

La progresiva desconexión de sistema y mundo de la vida es condición necesaria para el tránsito desde las sociedades estratificadas del feudalismo europeo a las sociedades de clases económicas de la modernidad temprana. [...] El patrón capitalista de modernización se caracteriza porque las estructuras simbólicas del mundo de la vida quedan deformadas, esto es, quedan cosificadas bajo los imperativos de los subsistemas diferenciados y autonomizados a través de los medios dinero y poder (p. 402).

La ruptura con el pasado es una de las características culturales de la modernidad. Lo que Habermas Ilama desconexión entre sistema y mundo de la vida en el tránsito entre feudalismo y capitalismo, Heilbroner (1972) lo expone en términos más convencionales:

El capitalismo se desarrolló a base de una población que, en gran parte, se había desprendido espiritual y físicamente de los inconvenientes feudales del Viejo Mundo, donde habían quedado rezagados muchos de los aspectos sociales y hábitos del pasado. Una conciencia de la posición de clase -y más aún, un reconocimiento explícito de la hostilidad entre las clases-, era tan notoria por su presencia en Europa como lo era en los Estados Unidos por su ausencia (p. 214).

El espíritu del capitalismo se encarna primero en los Estados Unidos antes de generalizar sus estructuras en el sistema económico internacional. No obstante, se puede encarnar porque antes ha colonizado la consciencia social; las dimensiones sociales y morales que Habermas reconoce como mundo de la vida.

Asimismo, la interpretación parsonsiana de la modernidad no ve en el aumento de complejidad y diferenciación de los sistemas desacoplamiento alguno, puesto que ha desestimado la categoría de mundo de la vida. Esto en busca de una potenciación de las posibilidades sistémicas a fin de reorganizar productivamente las actividades humanas, distribuidas en sistemas distintos, a saber, que se han diferenciado entre sí: "el aumento de la complejidad del sistema significa eo ipso un progreso en la dimensión de la configuración racional de las formas de vida" (Habermas, 1999, p. 407). 
En línea con lo anterior, la tesis de Max Weber (2013) acerca del desencantamiento del mundo se refiere al efecto modernizador de la racionalidad: la modernidad es un mundo desencantado porque es un mundo racionalizado, que ha perdido la fe en los encantamientos; en otras palabras, ha secularizado sistemáticamente sus estructuras redefiniéndolas racionalmente, pero con arreglo a fines. La racionalización del mundo moderno es obra de la racionalidad formal o instrumental, que atiende a los medios más eficaces para la consecución de fines; esa racionalidad se asienta, desplegándose en las estructuras sistémicas.

Al respecto, Habermas considera que el mundo de la vida está sustentado por otra racionalidad: la de los valores, que provee el sentido de las acciones. Ya Weber (2013) había planteado que la racionalización formal o sistémica del mundo se traduce en pérdida de sentido y libertad individual, tesis con la que Habermas concuerda. Parsons, en cambio, la discrepa:

Si las sociedades modernas desarrolladas se caracterizan por una alta complejidad y si, por lo que hace a las dimensiones capacidad de adaptación, diferenciación de subsistemas regidos por medios, inclusión y generalización de valores, la complejidad sólo puede aumentar en todas cuatro a la vez, entonces se da una relación analítica entre la alta complejidad del sistema, por un lado, y las formas universalistas de integración social y el individualismo flexiblemente institucionalizado, por otro. Es este esquema analítico el que obliga a Parsons a proyectar una imagen armónica de todo aquello que cae bajo la descripción de sociedad moderna (Habermas, 1999, p. 414).

El estructural funcionalismo parsonsiano asume con esto la función de legitimación ideológica del orden establecido. Para él, la racionalización moderna es buena, porque potencia el sentido y la libertad antes que generar anomia, que implica sujeción. La legitimación es una función sistémica que responde al imperativo de la autoconservación.

En la transferencia de la autonomía subjetiva a la sistémica, Parsons no observa reducción, sino po- tenciación: el individuo moderno contaría con más recursos para dedicarse a sus intereses particulares. El estado de autonomía sistémica llega a la sociedad por evolución natural de la complejización y diferenciación.

El contenido positivista de la teoría parsonsiana de sistemas tiende a naturalizar los hechos sociales en calidad de realidades dadas que no merecen cuestionarse: el conocimiento se limita a constatarlas mediante la descripción, sin pretender normarlas, porque la normatividad es intrínseca al propio funcionamiento sistémico. Los sistemas funcionan normalmente como están dados. Cuando se pretende alterar su funcionamiento normal desde una normatividad artificiosa, producto de la especulación subjetiva, se inducen disfunciones sistémicas.

Parte del contenido legitimador que asume el discurso parsonsiano es la representación de la evolución social de los países desarrollados como destino universal para todas las naciones. Habermas acepta que la teoría de la evolución social explica la especificidad de las naciones desarrolladas de la Modernidad, pero no concuerda en que esa configuración se reproduzca naturalmente en todo ámbito sociohistórico. La cultura moderna no es ejemplar ni destino apodíctico, sino ilustrativa de una de las posibles concreciones históricas de la acción humana.

La modernización, por su parte, es un proyecto concebido para adecuar el desarrollo de las naciones pobres a las pautas históricas que siguieron las que hoy son ricas. Pero en esa pretensión, desconoce la diversidad y especificidad de sociedades cuyas condiciones materiales las particularizan.

El proyecto hegemónico de la modernización procura globalizar la reproducción sistémica con base en generalizar la acción racional con arreglo a fines en todas las esferas de la acción humana. En este sentido, la condición necesaria para esa generalización es colonizar el mundo de la vida e inducir la racionalidad instrumental en la acción comunicativa; esto consiste en reducir la comunicación a coordinación lingüística de directrices funcionales: hacer de la comunicación un simple medio de control. 
La teoría de la acción comunicativa de Habermas comprende la comunicación como control, pero solo en una dimensión específica de un amplio espectro. En su mayor alcance, la comunicación, antes que la producción, es la interacción humana que mejor proyecta las potencialidades emancipatorias de la sociedad humana. La producción objetiva contiene bases que son condiciones materiales de emancipación: liberación de necesidades y autorrealización. No obstante, la comunicación determina el uso de esas bases.

De igual manera, Habermas contrasta el optimismo parsonsiano hacia el capitalismo moderno con la situación de la conciencia subjetiva en la modernidad tardía. La racionalización instrumental del mundo y el despliegue de la acción subjetiva al interior de sistemas sociales así racionalizados tiene por efecto la alienación de la conciencia subjetiva, que obstruye en principio cualquier autorrealización.

La racionalización formal coordina la acción en términos de función -categoría que se traslada de la acción sistémica al ámbito de la vida privada-, o mundo de la vida, en donde las acciones responden a la coordinación de la racionalidad sustantiva o con arreglo a valores. Es decir, el individuo nunca deja de ser funcionario: arrastra esa conciencia fuera de su espacio de integración funcional.

El individuo es una producción de la modernidad. La conciencia del agente como individuo se debe a la progresiva diferenciación que sigue a la desintegración de la conciencia premoderna, que en la cotidianeidad medieval se expresaba como conciencia de la pertenencia a una comunidad religada por la tierra-patria y la iglesia. La racionalización moderna individualiza a los actores en función de necesidades sistémicas, porque los sistemas requieren unidades individuales que asuman funciones estructurales. En Parsons, el individuo es una entidad que se diferencia como sistema psicosomático; fácticamente no expresa sino acciones que son funciones sistémicas, aunque desde las normas del sistema cultural esté llamado a la autorrealización personal.

En esta línea, Parsons (1966) concibe esa autorrealización dentro de la acción funcional, donde el indi- viduo encuentra condiciones de autonomía y libertad, es decir, no hay trascendencia más allá de los límites sistémicos. Para Habermas, esto no es sino la articulación sistémica de la represión individual: la inhibición de acciones que vayan más allá de la prescripción funcional, con pretensión emancipatoria, a saber, realización de las posibilidades y capacidades que la propia organización sistémica de la sociedad produce a la vez que reprime o libera restrictivamente.

Un ejemplo de esta denuncia habermasiana en torno a la reducción represiva de la prescripción funcional del lenguaje lo encontramos en la denominada "sociedad del conocimiento", que es un estado condicionado por el desarrollo de las ciencias y tecnologías de la información, que torna accesible e inmediato el patrimonio cultural de la humanidad, compuesto por ciencias, artes y letras. Pero lo hace bajo la categoría de información, desorganizada, saturadora y mezclada con producción cultural de calidad ínfima. La información no es conocimiento, por lo que, en realidad, lo que hay es una sociedad de la información, sin que se generalicen los instrumentos intelectuales para interpretarla y organizarla significativamente.

Desde el sistema cultural, se predica el valor de la individualidad en función de la autorrealización personal, pero desde el sistema social, la individualidad no es sino condición de integración funcional. Esta paradoja no se resuelve sino a base de romper el dualismo entre el sistema cultural normativo y los demás sistemas.

La renuencia de la teoría de sistemas a la normatividad extrasistémica se expresa en Parsons (1984) en su argumento de que las normas están dadas por el sistema de la cultura, que es el lugar de la validez no empírica. Las normas y valores culturales son ya sistémicos, porque la cultura se concibe desde esa categoría; no obstante, como hemos visto, se trata de una normatividad potencialmente conflictiva con la normatividad contenida en los demás sistemas, que responde a la autoconservación.

Habermas propone eliminar el dualismo entre facticidad y validez, pues considera que los fenómenos como la cultura, la sociedad y la personalidad son 
componentes que se expresan como mundo de la vida y como sistema. Los sistemas son hechos del mundo de la vida -siempre lo han sido-, aunque en la modernidad tardía se hayan diferenciado hasta constituirse en plexos autónomos de acción autorreferencial. Sin embargo, si se interpreta la sociedad como mundo de la vida - desde la experiencia espontánea cotidiana y la acción comunicativa- o se le concibe como sistema -desde la experiencia funcionalmente organizada por la razón instrumental-, entonces, la normatividad que desde la Teoría Crítica se pretende inducir al sistema no es propiamente ajena.

La alienación o enajenación de la modernidad tardía consiste en el posicionamiento del sistema como realidad fáctica ajena, enfrentada al mundo de la vida abocada al proceso de colonización de este mundo. Por consecuente, es la alienación de la racionalidad instrumental versus la racionalidad que sustenta la acción comunicativa, orientada al proceso de racionalización instrumental de todas las esferas de la acción humana.

La ascendencia sistémica de la racionalidad formal, que atiende exclusivamente a los medios eficaces para la consecución de fines, se evidencia irracional por la exclusión del análisis de la validez de los fines procurados. Es irracional por irreflexiva, ya que se concreta en una práctica tecnocrática antiintelectualista que concibe el conocimiento como poder, en tanto que hace del conocimiento técnico o científico un medio para la maximización de resultados. Con esto, se rehúsa ampliar la dimensión del conocimiento más allá de su aplicación práctica inmediata como medio de control.

Si la sociedad es una sola, lo es también la unidad de sistema y mundo de la vida, de razón instrumental y de razón sustantiva, de facticidad y de validez. El desacoplamiento tardo-moderno de sistema y mundo de la vida es consecuencia de la racionalización formal del mundo, que expresa la alienación de la razón moderna. Una de las ilusiones que produce la razón alienada es el dualismo entre facticidad y validez, entre un mundo de hechos con otro de valores. Tal dualismo lleva a Parsons (1984) a insistir, desde una teoría de la acción mal integrada, en la autonomía no empírica del sistema cultural, como en el pasado indujo en Weber la ilusión de la neutralidad axiológica de la ciencia.

Concebida, desde el estructural funcionalismo, como subsistema diferenciado y cerrado, la ciencia aparece ante la conciencia como sistema autónomo autorreferencial, con la producción de conocimientos como función específica. Desde esa perspectiva, a la ciencia le compete formular juicios de hecho, al margen de valoraciones, que corresponden a otro subsistema. Desde la perspectiva no dualista de la complementariedad diferenciada entre sistema y mundo de la vida, el mundo de los valores preexiste a la actividad científica y no la abandona, aun cuando los científicos no se aperciban de esa presencia. En este sentido, que la ciencia no esté realmente libre de valores significa que responde a intereses, pero también, lo hace diferenciadamente; lo que posibilita el orden social es la integración institucional entre valores e intereses.

Los intereses del conocimiento científico son intereses humanos, que se corresponden con los fenómenos que interesan a la ciencia y, por tanto, a quienes la practican. La diversidad de intereses científicos diversifica el sistema de la ciencia en saberes científicos. Habermas propone distinguir estos saberes por sus fenómenos, valores e intereses. A la distinción que hacía Dilthey (1974) entre ciencias de la naturaleza y ciencias del espíritu, añade una tercera categoría: las ciencias naturales, o empírico-analíticas (tales como: la física, la química, la medicina) están regidas por un interés técnico, sustentado por el valor de controlar fenómenos y procesos naturales, valor que puede sintetizarse como dominación; las ciencias sociales, o hermenéuticas (por ejemplo: la historia, el derecho, la sociología...) están regidas por un interés práctico, que concreta el valor de la comprensión o comunicación; las ciencias críticas (el psicoanálisis, la teoría social crítica, entre otras) están regidas por un interés emancipatorio, que concreta el valor de la libertad, esto es, la liberación subjetiva respecto de estructuras sociales opresoras (Habermas, 1992).

Etimológicamente, la palabra interés alude a una vinculación intersubjetiva: inter-esse es un núcleo 
que conecta a dos entes. Pero lo que realmente otorga al interés su dimensión intersubjetiva es su integración con los valores, que es función de las instituciones:

La orientación del agente en función de órdenes legítimos no puede excluir la orientación en función de sus propios intereses. [...] En la medida en que en los órdenes legítimos, los «fines últimos» o valores quedan puestos en relación, de manera ya selectiva, con los intereses existentes y se los hace compatibles con ellos, la acción institucionalizada puede ser entendida como un proceso de realización de valores en condiciones fácticas (Habermas, 1999, p. 295).

Normas, valores, intereses y acciones no son elementos desvinculados, porque son componentes de los procesos de integración social y funcional que se dan desde los espacios del mundo de la vida y de los sistemas. Entonces, las normas y los valores "preexisten" a las acciones, pero no en un mundo inteligible, sino en el bagaje experiencial del mundo de la vida, que es inmanente a la experiencia humana.

De lo anterior se desprende que, según Habermas, la normatividad de la teoría crítica es tanto expresión del mundo de la vida como de los sistemas; aunque la racionalidad que los sustenta en la modernidad contemporánea la desconozca. Esta normatividad o validez es consubstancial, porque precede a la actividad humana, la acompaña, la transforma y se transforma, como parte del mundo de la vida que se concreta en hechos. Las disfunciones no provienen de una normatividad artificiosamente inducida en los sistemas autónomos, sino de la recalcitrancia de los sistemas a reconocer la legitimidad y necesidad de esas normas.

La autorreferencia de los sistemas no es reflexividad, más bien significa opacidad, porque es pseudo-autorreferencia. Solo desde la apertura del sistema al mundo de la vida puede la sociedad devenir reflexiva o autorreflexiva, puesto que el ámbito de la validez, o del deber, es la auténtica autorreferencia, ya que es el contraste de la sociedad consigo misma: de la facticidad de la acción sistémica con la validez del mundo de la vida.
La estrategia para superar el dualismo teórico y práctico entre validez y facticidad, mundo de la vida y sistema, consiste en reacoplar el mundo de la vida al sistema. Este reacoplamiento expresa el problema de la teoría social, que comprende la oposición entre el mundo de la vida y los sistemas, con la tendencia colonizadora de estos. La teoría social ha de versar con la articulación adecuada de dos vías para el entendimiento intersubjetivo, denominadas 'sistema' y 'mundo de la vida', cuyo planteamiento Habermas (1999) esboza en los siguientes términos:

Un concepto provisional de sociedad como un sistema que tiene que cumplir las condiciones de mantenimiento propias de los mundos socioculturales de la vida. La fórmula de que las sociedades son plexos de acción sistémicamente estabilizados de grupos integrados socialmente precisa, qué duda cabe, de una explicación más detallada; por de pronto sólo representa la propuesta heurística de entender la sociedad como una entidad que en el curso de la evolución se diferencia lo mismo como sistema que como mundo de la vida. La evolución sistémica se mide por el aumento de la capacidad de control (Steuerungskapazitat) de una sociedad, mientras que la separación de cultura, sociedad y personalidad constituye un indicador del estado evolutivo de un mundo de la vida cuya estructura es una estructura simbólica (p. 215).

Ambas perspectivas son necesarias para la teoría y la práctica social. Por una parte, como perspectivas conceptuales, el sistema y el mundo de la vida explican procesos que ocurren en la vida real: la integración funcional y la integración social. Por otra parte, como perspectivas de la sociedad, el sistema y el mundo de la vida aluden a formas de actuar en el mundo. Su desacoplamiento moderno es un efecto patológico de la evolución social por incremento de complejidad y diferenciación.

Por tanto, la orientación generalizada de la acción por el sistema repercute en crisis diversas porque desintegra el mundo de la vida, con el efecto de la pérdida de sentido, que se traduce en anomia social. La comunicación se hace menos flexible, se empobrece y se fragmenta porque se orienta cada 
vez menos hacia el logro del consenso. La racionalización moderna, centrada en la razón funcional, diferenció y especializó sistemas, potenciándolos como máquinas de eficacia productiva y administrativa. En esa medida, se desacoplaron del mundo de la vida y se enfrentaron a él.

Concordamos con Habermas en que el mundo de la vida también debe incrementar su racionalización, pero con la orientación que le es específica: la razón con arreglo a valores, que se expresa en la acción comunicativa. La racionalización formal que invade el mundo de la vida, antes que reforzarlo, lo debilita en la consecución de su propósito, que es la comprensión y el sentido de la acción. Entonces, si Habermas apuesta por el reacoplamiento, es porque considera que ambas perspectivas, sistema y mundo de la vida, son complementarias, dado que comprenden dos expresiones complementarias de la realidad social.

La sociedad, actuando como mundo de la vida produce sentido y comprensión; y actuando como sistema, produce función y rendimiento, que redundan en autoconservación. El mundo de la vida es la sociedad reflexiva: su dimensión interna. El sistema es su proyección. Como perspectivas conceptuales, aluden a dos puntos de vista distintos, pero no incompatibles; el sistema es el punto de vista del observador externo, que describe objetivamente lo que ve. Esta es la perspectiva de la explicación (Erklären), que Dilthey (1974) juzgaba propia de las ciencias de la naturaleza. Se debe mencionar que Marvin Harris (1988), desde otra tradición teórica, la denomina Etic. En síntesis, el mundo de la vida es el punto de vista de quien está dentro y describe subjetivamente lo que experimenta, asumiendo la perspectiva del entendimiento o comprensión (Verstehen) o del método emic.

El desacoplamiento asimétrico, no obstante, pone en riesgo la misma autoconservación, porque las funciones tienden a la pérdida de sentido: aún más, conllevan efectos destructivos sobre la misma realidad material de la que depende la autoconservación. El fortalecimiento de la racionalización sustantiva del mundo de la vida tiene el horizonte de la integración de ambas expresiones de la realidad social, así como de la misma racionalidad. El potencial de un compromiso del sistema con la integridad del mundo de la vida y de este con la especificidad funcional de aquel proyecta un estadio cualitativamente diferente de la evolución social, con un incremento en la complejidad que no se traduce en diferenciaciones asimétricas, sino recíprocamente enriquecedoras.

La eficacia productiva y administrativa del sistema, complementada con la racionalidad sustantiva del mundo de la vida, reincorporaría el sentido a la acción funcional, a la vez que corregiría los efectos destructivos de esa acción sobre los recursos materiales. Reacoplar ambas perspectivas no solo pondría garantías a la autoconservación, sino que dignificaría la condición humana y la potenciaría para la autorrealización en nuevas formas de evolución social.

En su análisis crítico, Habermas demuestra que Parsons utiliza las categorías para elaborar un discurso social y políticamente conservador. Bajo la apariencia de objetividad, el funcionalismo, en la versión de Parsons (1984), contribuye a la legitimación de estructuras de dominación, cuyas condiciones de producción, así como sus posibilidades alternativas, pueden visibilizarse empleando las mismas categorías-complejidad y sistema-con las que esa teoría ideológica pretende garantizar la funcionalidad sistémica. Al respecto, Habermas evidencia que esa funcionalidad es aparente, ya que en realidad los sistemas sociales vigentes son profundamente disfuncionales, porque perpetúan aberraciones -pobreza material, opresión política, injusticia, ignorancia, alienación social, deterioro ambiental- que son factiblemente superables.

La aspiración de mejorar la condición humana no es un ideal ilusorio. Implica identificar los fines junto con las estrategias indispensables para alcanzarlos, yace en la integración complementaria del mundo de la vida -que es fuente y meta del sentido intersubjetivo- y del sistema -que constituye garantía instrumental en la prosecución de fines pragmáticos. Sin embargo, esa necesaria integración demanda transformaciones en los ámbitos del pensamiento y de la acción. Es decir, en la visión de 
mundo, en su posible ilustración, en la sensibilidad que deriva de esa visión, así como en las interacciones subjetivas, prácticas sociales, o modos de ser, que son efecto y factor de esa visión.

\section{REFERENCIAS BIBLIOGRÁFICAS}

Dilthey, W. (1974). Teoría de las concepciones del mundo. Madrid: Revista de Occidente.

Ferrater-Mora, J. (1998). Diccionario de Filosofía. Barcelona: Ariel.

Giddens, A., Turner, J., Alexander, J. C., Homans, G. C., Joas, H., Münsch, R. \& Honneth, A. (1990). La teoría social, hoy. México: Alianza.

Habermas, J. (1999). Teoría de la acción comunicativa, II. Sobre la crítica de la razón funcionalista. Madrid: Taurus.

Habermas, J. (1992). Conocimiento e interés. Madrid: Taurus.

Habermas, J. (1993). El discurso filosófico de la modernidad. Madrid: Taurus.

Harris, M. (1988). Introducción a la antropología general. Madrid: Alianza.
Heilbroner, R. L. (1972). La formación de la sociedad económica. México: FCE.

Horkheimer, M. (1973). Crítica de la razón instrumental. Buenos Aires: Editorial Sur.

Husserl, E. (2008). La crisis de las ciencias europeas y la fenomenología trascendental. Buenos Aires: Prometeo Libros.

Izuzquiza, I. (2008). La Sociedad sin hombres. Niklas Luhmann o la teoría como escándalo. Barcelona: Anthropos.

Luhmann, N. (2010). ¿Cómo es posible el orden social? Barcelona: Herder.

Parsons, T. (1966). Societies. Evolutionary and Comparative Perspectives. N.J.: Prentice Hall.

Parsons, T. (1984). El Sistema social. Madrid: Alianza.

Pizzi, J. (2005). El mundo de la vida. Husserl y Habermas. Santiago: Ediciones UCSH.

Weber, Max (2013). Ensayos sobre metodología sociológica. Buenos Aires: Amorrortu. 